OPEN ACCESS

Edited by:

Jianping Wang,

University of Florida, United States

Reviewed by:

Muthu Venkateshwaran,

University of Wisconsin-Platteville,

United States

Dong Wang,

University of Massachusetts Amherst,

United States

Keiko Yoshioka,

University of Toronto, Canada

*Correspondence:

B. W. Poovaiah

poovaiah@wsu.edu

Specialty section:

This article was submitted to

Plant Microbe Interactions,

a section of the journal

Frontiers in Plant Science

Received: 15 June 2017

Accepted: 24 October 2017

Published: 16 November 2017

Citation:

Jauregui $E, D u$ L, Gleason $C$ and

Poovaiah BW (2017) W342F

Mutation in CCaMK Enhances its

Affinity to Calmodulin But

Compromises Its Role in Supporting

Root Nodule Symbiosis in Medicago

truncatula. Front. Plant Sci. 8:1921.

doi: 10.3389/fpls.2017.01921

\section{W342F Mutation in CCaMK Enhances Its Affinity to Calmodulin But Compromises Its Role in Supporting Root Nodule Symbiosis in Medicago truncatula}

\author{
Edgard Jauregui', Liqun Du ${ }^{1,2}$, Cynthia Gleason ${ }^{3}$ and B. W. Poovaiah ${ }^{1 *}$ \\ ${ }^{1}$ Laboratory of Molecular Plant Science, Department of Horticulture, Washington State University, Pullman, WA, \\ United States, ${ }^{2}$ College of Life and Environmental Sciences, Hangzhou Normal University, Hangzhou, China, ${ }^{3}$ Department of \\ Plant Pathology, Washington State University, Pullman, WA, United States
}

The calcium/calmodulin-dependent protein kinase (CCaMK) is regulated by free $\mathrm{Ca}^{2+}$ and $\mathrm{Ca}^{2+}$-loaded calmodulin. This dual binding is believed to be involved in its regulation and associated physiological functions, although direct experimental evidence for this is lacking. Here we document that site-directed mutations in the calmodulinbinding domain of CCaMK alters its binding capacity to calmodulin, providing an effective approach to study how calmodulin regulates CCaMK in terms of kinase activity and regulation of rhizobial symbiosis in Medicago truncatula. We observed that mutating the tryptophan at position 342 to phenylalanine (W342F) markedly increased the calmodulin-binding capability of the mutant. The mutant CCaMK underwent autophosphorylation and catalyzed substrate phosphorylation in the absence of calcium and calmodulin. When the mutant W342F was expressed in ccamk-1 roots, the transgenic roots exhibited an altered nodulation phenotype. These results indicate that altering the calmodulin-binding domain of CCaMK could generate a constitutively activated kinase with a negative role in the physiological function of CCaMK.

Keywords: $\mathrm{Ca}^{2+} /$ calmodulin-dependent protein kinase, calcium, calmodulin, symbiosis, Medicago truncatula

\section{INTRODUCTION}

Calcium/calmodulin $\left(\mathrm{Ca}^{2+} / \mathrm{CaM}\right)$-mediated signaling plays important roles in sensing and transducing environmental stimuli and developmental cues (Du and Poovaiah, 2005; Poovaiah et al., 2013), plant immune responses (Du et al., 2009) and mutualistic plant-microbe interactions (Gleason et al., 2006; Yuan et al., 2017). One important effector protein of this $\mathrm{Ca}^{2+} / \mathrm{CaM}$ mediated signaling in plants is the $\mathrm{Ca}^{2+} / \mathrm{CaM}$-dependent protein kinase, CCaMK (Patil et al., 1995). CCaMK is nuclear localized and lies downstream of the cellular calcium spiking response that occurs in the symbiosis pathway. After activation by $\mathrm{Ca}^{2+} / \mathrm{CaM}$, CCaMK phosphorylates CYCLOPS or IPD3 in Medicago truncatula, which are required for symbiosis (Yano et al., 2008).

CCaMK has been well studied not only because of its unique protein structure, but also due to its critical role in the symbiotic interaction between leguminous plants and bacteria and/or fungi (Patil et al., 1995; Ramachandiran et al., 1997; Harper et al., 2004). The protein structure of CCaMK 
contains a catalytic domain followed by a CaMbinding/autoinhibitory domain and three EF hand motifs (Sathyanarayanan et al., 2001; Sathyanarayanan and Poovaiah, 2002; Singh and Parniske, 2012). CCaMK is regulated by free $\mathrm{Ca}^{2+}$ which binds to the EF hand motifs and $\mathrm{Ca}^{2+}$-loaded CaM, which binds to the autoinhibitory domain (Lévy et al., 2004; Tirichine et al., 2006; Pumplin et al., 2010). This two-step regulatory mechanism allows CCaMK to become active and promote phosphorylation of target proteins that regulate root nodule symbiosis and mycorrhizal arbuscule development (Wang and Poovaiah, 1999; Sathyanarayanan and Poovaiah, 2002; Parniske, 2004). Both regulatory domains are important during symbiotic response (Tirichine et al., 2006; Pumplin et al., 2010). Previous reports demonstrated that expressing only the kinase domain (KD) of this protein or expressing a mutant of the threonine at position 271 (Thr271), located in the $\mathrm{KD}$, resulted in the formation of spontaneous nodules in the absence of bacteria in Medicago roots (Sathyanarayanan et al., 2001; Gleason et al., 2006; Tirichine et al., 2006). This indicates that CCaMK's autoinhibitory domain and the EF-hand motifs negatively regulate the kinase activity. Another report has also shown the importance of the EF hand motifs in negative regulation of CCaMK. This study indicated that the EF hand motifs bind free $\mathrm{Ca}^{2+}$ and keep the CCaMK inactive at basal $\mathrm{Ca}^{2+}$ concentrations (Shimoda et al., 2012). Conversely, if free $\mathrm{Ca}^{2+}$ is blocked from binding to the EF hand motifs, CCaMK becomes auto-activated (Hayashi et al., 2010; Miller et al., 2013). Recent reports have demonstrated that CCaMK might be regulated by DELLA proteins (Jin et al., 2016) and/or TOR (the target of rapamycin) protein kinase (Nanjareddy et al., 2016) which are essential for symbiotic rhizobial pathway. Furthermore, CCaMK gene is very well conserved among phytozome species (Wang et al., 2015). CCaMKs have been found in peanuts (Arachis hypogaea) and tomato (Solanum lycopersicum), where it may play roles in the symbiotic pathway (Peng et al., 2017) and/or disease resistance (Wang et al., 2015).

Generally, the key to the activation of the kinase requires the release of the protein's own autoinhibition, which can be accomplished by the binding of CaM to the autoinhibitory/CaMbinding domain (Hayashi et al., 2010; Antolín-Llovera et al., 2012). In 2011, researchers using an auto activated form of CCaMK concluded that CaM-binding is essential for nodulation development during rhizobial symbiosis, but it is not required for fungal symbiosis (Horváth et al., 2011). However, since this auto-activated form could no longer interact with either CaM or free $\mathrm{Ca}^{2+}$, it is critical to study the relevance of the CaMbinding alone during the activation of CCaMK (Takeda et al., 2012). Furthermore, CCaMK protein structure is unique to plants and understanding of its activation and regulation is far from clear. There are reports which have focused in its $\mathrm{KD}$ and visinin-like domain and have determined their importance of specific amino acids that regulate its kinase activity in addition to calmodulin and $\mathrm{Ca}^{2+}$ binding. Studies on the relationship between calmodulin and $\mathrm{Ca}^{2+}$ binding and kinase activity could reveal how this kinase interprets the $\mathrm{Ca}^{2+}$ signature during the establishment of plant-microbe symbioses.
In this study, we used a site-directed mutagenesis approach to alter the Calmodulin-binding/autoinhibitory domain of CCaMK in order to observe any variation of the CaM-binding capacity of this protein kinase in the presence of $\mathrm{Ca}^{2+}$. We generated a series of mutations at a critical residue in the autoinhibitory domain (Trp-342) and measured the effects of the mutations on CaM-binding capacity. We found that the mutant $\mathrm{W} 342 \mathrm{~F}$ has increased CaM-binding capacity, even in the absence of $\mathrm{Ca}^{2+}$. Interestingly, roots expressing W342F developed nodules after rhizobial inoculation, but half of these nodules were smaller than normal and were poorly colonized by bacteria.

\section{MATERIALS AND METHODS}

\section{Site-Directed Mutagenesis}

Site-directed mutagenesis of CCaMK was performed using a high fidelity KOD DNA polymerase enzyme to construct the desired plasmid with the mutations, following protocol as described (Liu and Naismith, 2008). The mutated CCaMK cds were confirmed by sequencing.

\section{Expression and Purification of CCaMK and Site-Directed Mutants}

The full-length of CCaMK and its site-directed mutants were cloned into the bacterial expression system pET28b and transformed into Escherichia coli strain BL21 (DE3)/pLysS. The bacteria carrying the above plasmids were grown in LB liquid media containing kanamycin at $37^{\circ} \mathrm{C}$ until $\mathrm{OD}_{600}$ of the culture reached 0.5 units. Once the liquid culture reached this optimal density, $0.5 \mathrm{mM}$ isopropyl $\beta$-D-1-thiogalactopyranoside (IPTG) was added to induce the recombinant protein. After 3$\mathrm{h}$ induction, cells were harvested and broken using lysozyme treatment $(1 \mathrm{mg} / \mathrm{ml})$ followed by sonication. The recombinant protein was purified with Ni-NTA agarose affinity beads (Qiagen) as described in the manufacturer's manual. The purified proteins were dialyzed against buffer containing $40 \mathrm{mM}$ Tris $\mathrm{pH}$ 7.6, $1 \mathrm{mM}$ dithiothreitol (DTT), 1 mM EDTA, and 10\% ethylene glycol. Dialyzed proteins were quantified by Bradford assay and stored at $-80^{\circ} \mathrm{C}$ with $15 \%$ glycerol.

\section{CaM-Binding Assays}

The CaM-2 from Arabidopsis conjugated with horseradish peroxidase (CaM-HRP) was used to study the CaM-binding property of CCaMK mutants. The induced proteins of CCaMK and its mutants in pET28b were separated by SDS-PAGE (15\%) and transferred onto PVDF membrane. The membrane was blocked in binding buffer $(10 \mathrm{mM}$ Tris $\mathrm{pH} 7.5,150 \mathrm{mM} \mathrm{NaCl}$, $1 \mathrm{mM} \mathrm{CaCl}_{2}$ ) containing 5\% non-fat dry milk for $1 \mathrm{~h}$ at room temperature, then incubated with milk containing binding buffer supplemented with AtCaM2-HRP (1:1000 dilution) for $1 \mathrm{~h}$ at room temperature. The membrane was then washed three times in binding buffer for $10 \mathrm{~min}$ each. To detect the CaM signal, the BM chemiluminescence Western blotting kit (Roche Applied Science) was used according to instructions from manufacturer. 


\section{Autophosphorylation Assays}

The autophosphorylation assay was performed in $10 \mu \mathrm{l}$ reaction mixture using $0.4 \mu \mathrm{g}$ of CCaMK protein and its mutated versions. The reaction buffer contained $50 \mathrm{mM}$ HEPES $\mathrm{pH} 7.5,10 \mathrm{mM}$ magnesium acetate, $1 \mathrm{mM}$ DTT, $10 \mu \mathrm{M}$ ATP and $0.5 \mu \mathrm{Ci} / \mu \mathrm{l}$ $\left[\gamma^{-32} \mathrm{P}\right]$ ATP, in the presence of $5 \mathrm{mM}$ EGTA with or without $1 \mu \mathrm{M}$ of bovine brain CaM (Sigma); and $0.5 \mathrm{mM}$ of $\mathrm{CaCl}_{2}$ with or without bovine CaM. Samples were incubated at $30^{\circ} \mathrm{C}$ for $30 \mathrm{~min}$. To stop the reaction, SDS-PAGE sample buffer was added, followed by boiling the samples for $2 \mathrm{~min}$. Samples were separated by a $12.5 \%$ SDS-PAGE. Protein gel was then dried and exposed to autoradiography film (Kodak).

\section{Substrate Phosphorylation Assays}

All of the in vitro kinase assays used $0.4 \mu \mathrm{g}$ of purified protein in a $10 \mu \mathrm{l}$ total volume. The buffer contained $50 \mathrm{mM}$ HEPES $\mathrm{pH}$ 7.5, $10 \mathrm{mM}$ magnesium acetate, $1 \mathrm{mM}$ DDT, $100 \mu \mathrm{M}$ ATP, and $0.5 \mu \mathrm{Ci} / \mu \mathrm{l}\left[\gamma^{-32} \mathrm{P}\right]$ ATP in the presence of $5 \mathrm{mM}$ EGTA or $0.5 \mathrm{mM} \mathrm{CaCl}_{2}$ or $0.5 \mathrm{mM} \mathrm{CaCl}_{2}$ with $1 \mu \mathrm{M}$ of bovine brain CaM (Sigma). In order to determine substrate phosphorylation, two micrograms of a bovine myelin basic protein (MBP) was used as substrate. Reactions were stopped by adding SDS-PAGE sample buffer and then boiled for $1 \mathrm{~min}$ in water bath. Subsequently, sample reactions were analyzed in SDS-PAGE and gel as dried. The difference of substrate phosphorylation patterns at different conditions was observed by exposing gel to the Kodak autoradiography film.

\section{Hairy Root Transformation}

The generation of transgenic roots was performed using a binary vector (pDL28-DPR) containing the native promoter of CCaMK and a red fluorescent protein encoding region from Discosoma sp. (DsRed) driven by Arabidopsis ubiquitin-10 promoter. The full-length CCaMK and mutant gene (W342F) were digested with SpeI and SalI restriction enzymes and ligated into the binary vector. The verified constructs were transformed into Agrobacterium rhizogenes strain K599 to transform the rootless plantlets of wild-type and ccamk-1 mutant of M. truncatula A17. The control groups used in this experiment were wildtype $M$. truncatula explants were transformed with the binary vector without any gene (empty vector). In the other control group, the ccamk-1 mutant plants were introduced with the empty vector. Our experimental groups were as follows: The third and fourth group used ccamk-1 plants which were transformed with the binary vector carrying the full-length CCaMK gene and $\mathrm{W} 342 \mathrm{~F}$ mutant gene, respectively. The seedlings were grown on buffered nodulation medium (BNM) agar plates with Kanamycin selection. After 3 weeks, the plants were screened for transgenic roots by using a Kodak imaging system (Model 4000MM) with corresponding filters for the expression of DsRed fluorescent protein. For the nodulation experiments, the plants with transgenic roots were inoculated with Sinorhizobium meliloti 2011 carrying a green fluorescent protein (GFP) reporter gene and transferred to sterilized growth pouches (Mega International). The inoculated seedlings were watered with $1 / 10$ dilution of nitrogen-deprived nutrient media (BNM) and grown under $16 / 8$-h light/dark cycle at $21^{\circ} \mathrm{C}$ for 28 days. At 28 -days post-inoculation, nodules were counted and observed under a Leitz fluorescent stereomicroscope using optical filters for GFP. Green fluorescence verified the presence of living rhizobia inside the developed nodule.

\section{Confocal Microscopy}

Selected nodules were analyzed by Zeta 510 Meta confocal microscope in order to determine the location of the bacteria in the nodules formed on the roots of ccamk-1 complemented with wild-type and mutated version of CCaMK (W342F). Nodules were excised in half and were stained with Calcofluor white, a special fluorescent stain that allows observation of cell walls of tissues due to its binding to cellulose and chitin. For this fluorescent stain, we used excitation filter of $365 \mathrm{~nm}$ and emission filter $420 \mathrm{~nm}$ in $10 \times$ microscope lenses. To determine GFP, we used excitation filter $380 \mathrm{~nm}$ and emission filter $480 \mathrm{~nm}$. Merged pictures were obtained and subsequently analyzed.

\section{RESULTS}

\section{A W342F Mutation in the CCaMK Autoinhibitory Binding Domain Can Positively Increase Its CaM-Binding Capacity}

The CaM-binding domain in CCaMK interacts with CaM, therefore, site-directed mutations were generated in the CaMbinding/autoinhibitory domain to investigate whether the CaM-binding capacity and kinase activity are correlated. Four residues in the calmodulin-binding/autoinhibitory domain were selected: F327, L333, I338, and W342. Mutations in the latter one produced some interesting results. W342 was mutated to five different residues with less mass but similar hydrophobic property (Figure 1A). These mutants were tested for CaM-binding capability. The CaM-binding assay showed that one mutant, W342F, exhibited increased CaM-binding capacity as compared to wild-type CCaMK. W342L and W342I showed less binding to CaM in comparison to CCaMK and W342F. The mutant W343V no longer interacted with CaM, however, W342A mutant was able to bind to CaM with a similar capacity as wild-type CCaMK (Figure 1B).

\section{Significance of W342F Mutant on Its Kinase Activity}

In CCaMK, the binding of CaM to the autoinhibitory domain releases its autoinhibition and activates its kinase activity. Since the W342F mutant showed an increase in CaM-binding ability, we hypothesized that the kinase activity of this mutant could be increased. Therefore, we specifically focused on this potential "gain of function" mutant, and an in vitro kinase assay was carried out.

Normally, the autophosphorylation of CCaMK is very low in the absence of $\mathrm{Ca}^{2+}$, and it increases drastically once $\mathrm{Ca}^{2+}$ is added to the reaction mixture. Similar to previously published reports, we found that the autophosphorylation of 
A

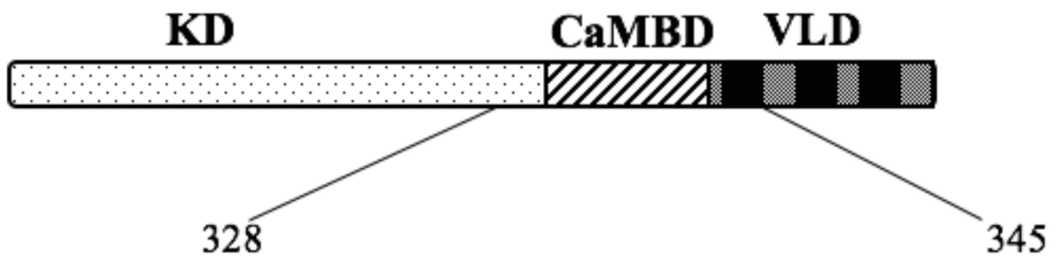

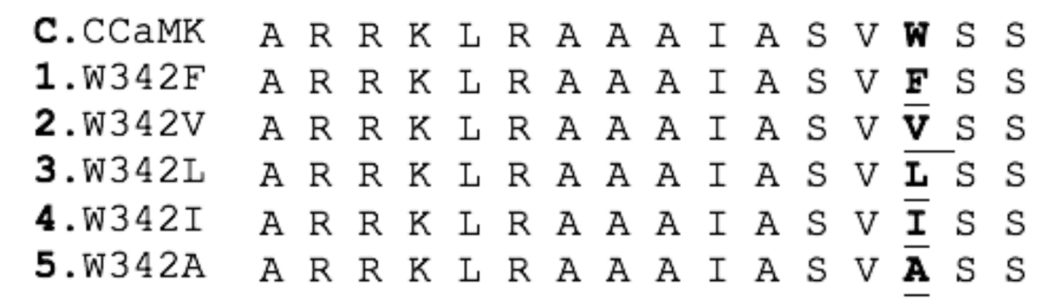

B

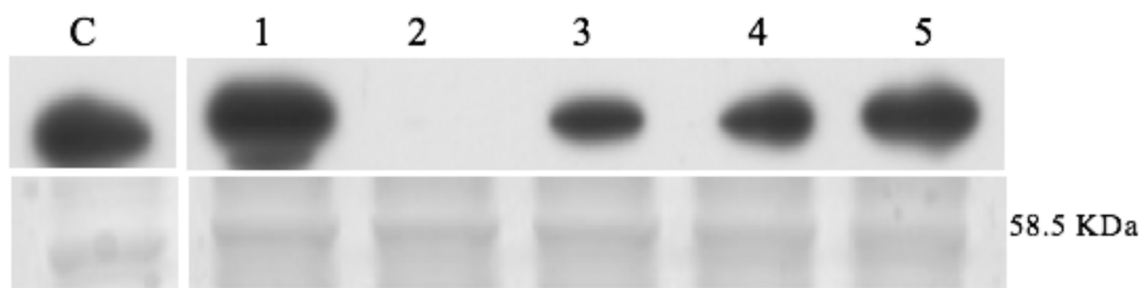

FIGURE 1 | Site-directed mutants and calmodulin-binding assay. Protein structure of CCaMK contains three domains: kinase domain (KD), calmodulin-binding/autoinhibitory domain (CAMBD) and visinin-like domains (VLD). Five mutants were generated by substituting the residue Trp-432 in the calmodulin-binding/autoinhibitory domain of CCaMK (A). For calmodulin-binding assay (B), total proteins from Escherichia coli (BL21) expressing the pET28-CCaMK or its mutated versions were separated on SDS-PAGE, transferred to PVDF membrane and incubated with horseradish peroxide (HRP)-conjugated CaM in the presence of $\mathrm{Ca}^{2+}(1 \mathrm{mM})$. Upper: Calmodulin binding overlay assay of wild-type and all 5 of the mutants of CCaMK as showed in "A"; Lower: Coomassie staining of proteins from E. coli (BL21) expressing the pET28-CCaMK or its mutated versions (protein size: $58.5 \mathrm{kDa}$ ). The CaM-binding assay was repeated at least 4 times to corroborate the difference.

the wild-type CCaMK was decreased after the addition of CaM (Takezawa et al., 1996; Miller et al., 2013). Interestingly we found the $\mathrm{W} 342 \mathrm{~F}$ mutant was autophosphorylated even in the absence of $\mathrm{Ca}^{2+}$. The W342F mutant was also autophosphorylated in the presence of CaM and $5 \mathrm{mM}$ EGTA. In the presence of $\mathrm{Ca}^{2+}$, both wildtype CCaMK and the W342F mutant underwent autophosphorylation. However, the autophosphorylation intensity of W342F mutant was reduced in comparison to wild-type CCaMK (Figure 2A and Supplementary Figures 1,2). It seems that the activation of autophosphorylation in $\mathrm{W} 342 \mathrm{~F}$ mutant is no longer dependent on $\mathrm{Ca}^{2+}$, on the contrary, it responds to the $\mathrm{Ca}^{2+}$ signal in an opposing manner as compared to wild-type CCaMK.

We used bovine MBP as substrate to test the substrate phosphorylation activities of CCaMK. These reactions were performed under three different conditions: (1) in the absence of $\mathrm{Ca}^{2+},(2)$ in the presence of $\mathrm{Ca}^{2+}$, and (3) in the presence of $\mathrm{Ca}^{2+}$ and CaM. The W342F mutant phosphorylated the substrate in all three conditions, with a slightly increased intensity in the presence of both $\mathrm{Ca}^{2+}$ and CaM. Nevertheless, in comparison to $\mathrm{CCaMK}$, this mutant showed a clear reduction in substrate phosphorylation activity even in the presence of $\mathrm{Ca}^{2+}$ and CaM (Figure 2B and Supplementary Figures 1, 2). Thus, in the W342F mutant substrate phosphorylation is $\mathrm{Ca}^{2+}$-independent and also CaM-independent, and barely responds to $\mathrm{Ca}^{2+}$ and CaM inputs.

\section{W342F Negatively Regulates Root Nodule Symbiosis}

With the clear indication that the phosphorylation activity of W342F mutant differs from wild-type CCaMK in its biochemical property, we wanted to test the effect of the $\mathrm{W} 342 \mathrm{~F}$ on nodule formation. The loss-of-function ccamk-1 Medicago plants were transformed with W342F or CCaMK by Agrobacterium rhizogenes transformation. The transformed roots were inoculated with $S$. meliloti 2011 tagged with a GFP. The ccamk-1 plants inoculated with $S$. meliloti 2011-GFP did not produce nodules. The ccamk-1 mutant complemented with wild-type CCaMK produced pink mature nodules at 28-days post-inoculation with S. meliloti 2011-GFP. Interestingly, at 28-days post-inoculation, the ccamk-1 plants transformed with $\mathrm{W} 342 \mathrm{~F}$ were able to produce nodules, indicating that the W342F mutant can complement the ccamk-1 plants (Figures 3, 4). The total number of nodules produced on the roots of ccamk-1 mutant complemented with the $\mathrm{W} 342 \mathrm{~F}$ mutant (ccamk-1 + W342F) was comparable to the number of nodules on roots of ccamk-1 mutant 


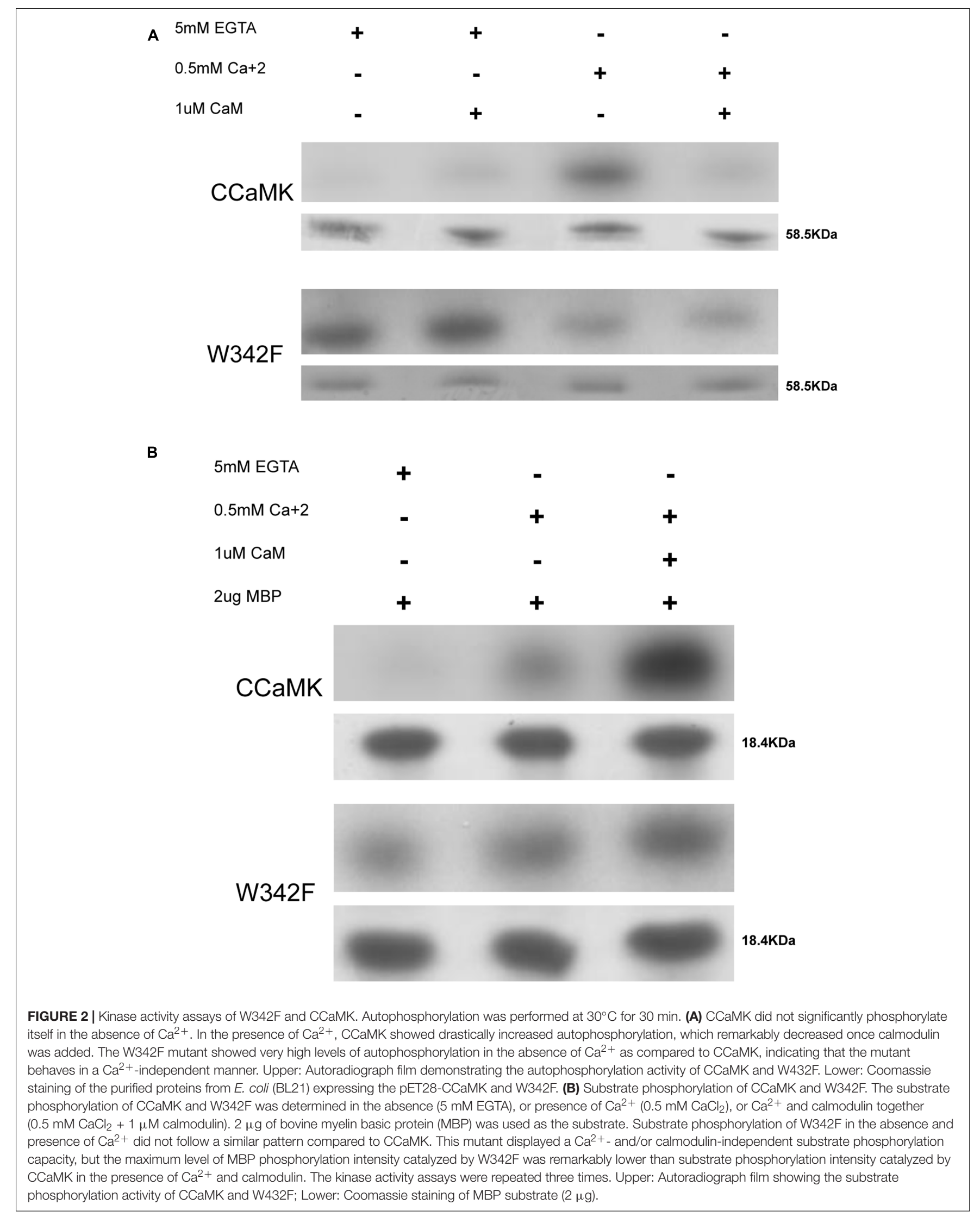



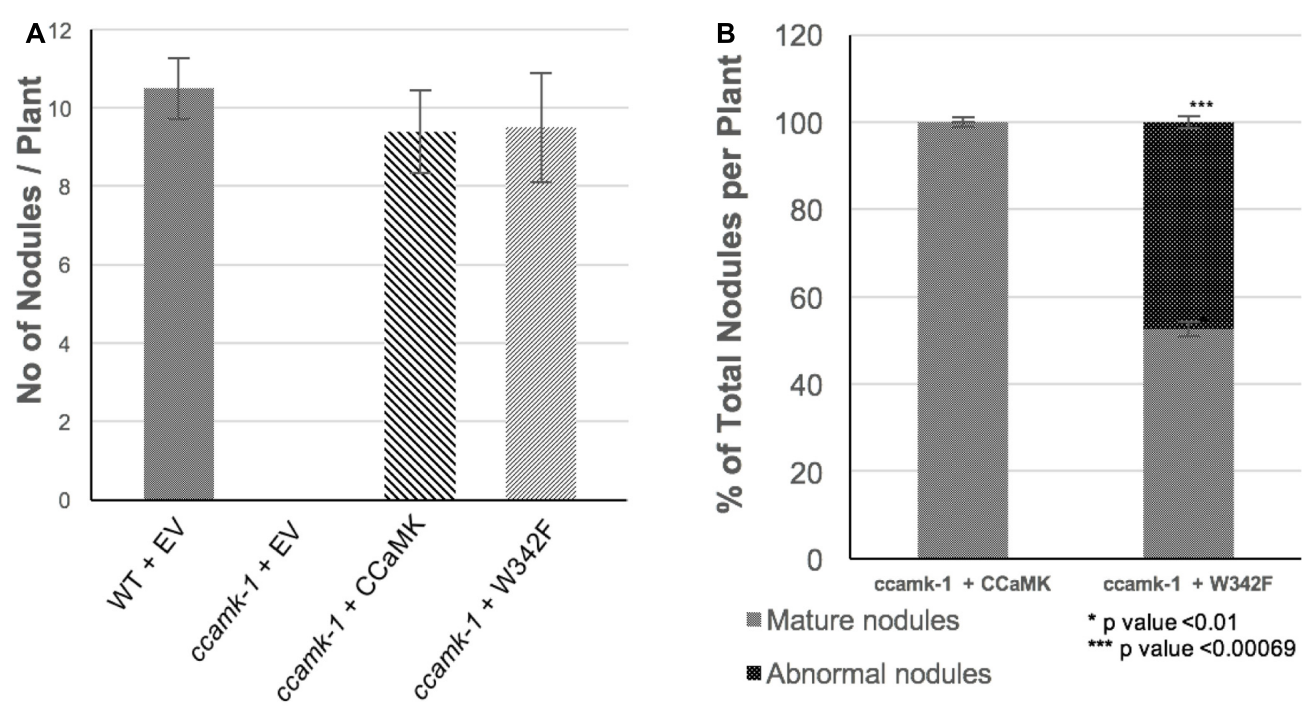

FIGURE 3 | The W342F mutant plants are able to form altered nodules. The roots of ccamk-1 mutant were transformed with the W342F mutant of CCaMK, roots of WT and ccamk-1 transformed with empty vector were used as positive and negative controls. (A) The number of nodules per plant for each construct was quantitated 28-days after inoculation with Sinorhizobium meliloti 2011-Green fluorescent protein (GFP). (B) Phenotypic difference of nodule development on ccamk-1 roots complemented with W342F and CCaMK. W342F mutation resulted in significant increase $(p$-value $=0.00069)$ in the number of small nodules.

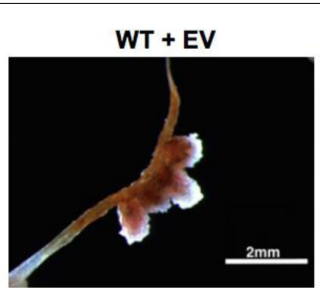

ccamk-1 + EV
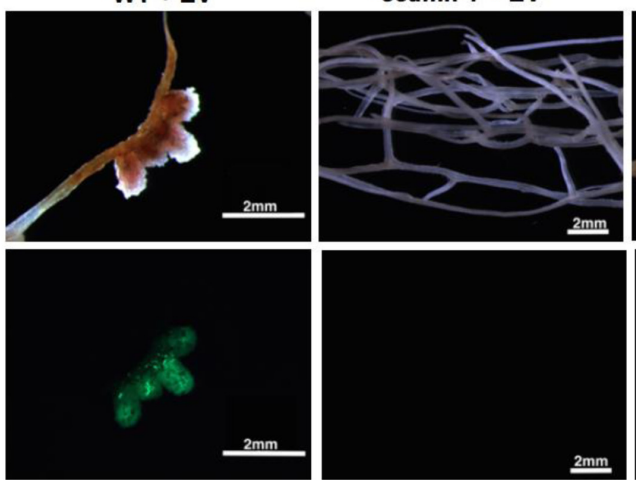

ccamk-1 + CCaMK
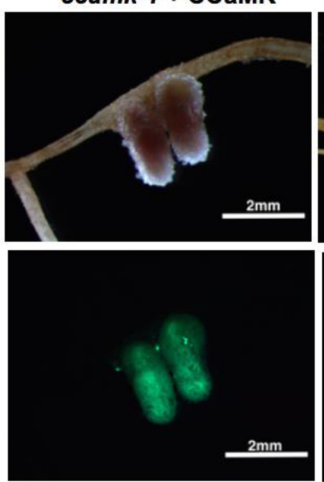

ccamk-1 + W342F
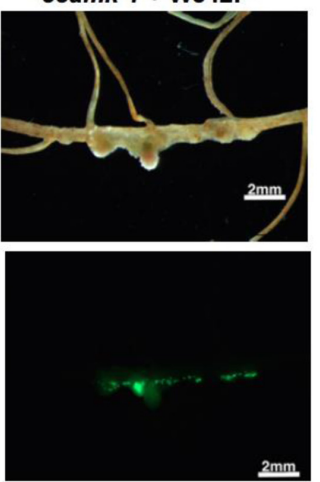

FIGURE 4 | W342F mutation resulted in altered nodule development. Root phenotypes were observed for each construct. Upper: Bright field picture. Lower: GFP fluorescent image of bacteria established in the nodule structure (green color). The W342F mutant complemented ccamk-1 for root nodule symbiosis.

complemented with wild-type CCaMK (ccamk-1 + CCaMK) (Figure 3A).

Although the total number of nodules was equivalent between ccamk-1 + CCaMK and ccamk-1 + W342F plants, the number of mature, pink nodules on the ccamk-1 + W342F plants roots was significantly less than that on the ccamk-1 + CCaMK roots $(p$-value $=0.01)$. The ccamk-1 + CCaMK roots produced $100 \%$ mature, pink nodules after inoculation with rhizobia. Approximately $50 \%$ of the nodules on the ccamk-1 + W342F plants were small abnormal nodules. These small nodules were not observed in the wild-type CCaMK inoculated with rhizobia $(p$-value $=0.00069)$ (Figure 3B). The abnormal nodules were white, indicating poor colonization by rhizobia (Figure 5A). Confocal microscopic analysis revealed that GFP-labeled S. meliloti 2011 was inside these small nodules but the distribution patterns of the bacteria in the nodule differed from that of the normal mature nodules (Figure 5B).

\section{DISCUSSION}

Extensive studies have been conducted by using site-directed mutagenesis as a powerful tool to manipulate the coding region of various genes. We took advantage of this wellknown strategy to modify a residue on the autoinhibitory/CaMbinding domain in order to alter its well-defined structure and modify the CaM-binding capacity of CCaMK. Altering the CaM-binding capacity of CCaMK should provide a better understanding of the regulation of CCaMK's autoinhibitory domain. 

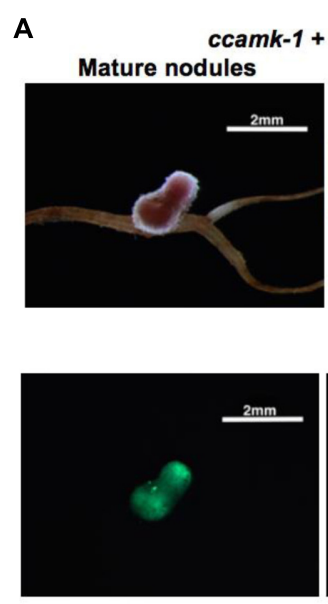

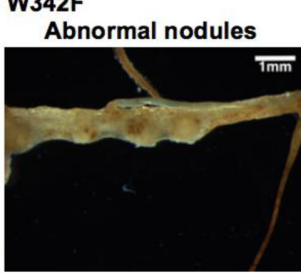

B

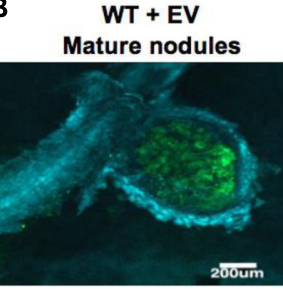

ccamk-1

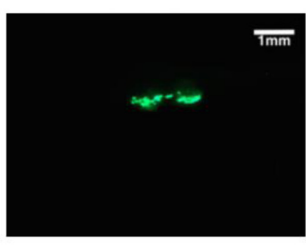

ccamk-1 + CCaMK Mature nodules

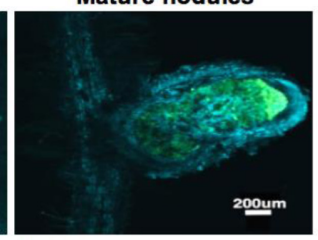

W342F

Abnormal nodules

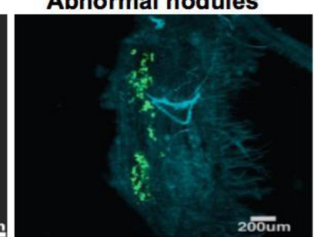

FIGURE 5 | (A) Morphological differences in nodules produced on roots complemented with W342F and CCaMK; (B) Confocal microscopic images of mature nodules from roots of wild-type Medicago truncatula and ccamk-1 knockout complemented with CCaMK and W342F mutant. Blue color indicates cell wall tissue stained with Calcofluor white. Green color shows living S. meliloti 2011.

The CaM-binding domain contains particular structural features: it forms an $\alpha$ helix with a net positive charge and two hydrophobic anchor residues on one side. These characteristics allow CaM to bind easily in the presence of $\mathrm{Ca}^{2+}$ (Yang et al., 2007). Typically, CaM contains two lobes at its $\mathrm{N}$ - and C-terminals that are connected by a helix linker, and in the presence of $\mathrm{Ca}^{2+}$, these two lobes will wrap the CaM-binding domain (Tidow and Nissen, 2013). However, there have been reports that some CaM-binding domains showed increased affinity to either of the terminals (Snedden and Fromm, 2001; Liu et al., 2012). This suggests that manipulating the $\alpha$ helix motif can modify its capacity to bind to CaM (Jang et al., 2011; Gifford et al., 2013).

One amino acid in the autoinhibitory/CaM-binding domain of CCaMK (W342) was replaced by amino acids with similar hydrophobicity but smaller masses in order to alter the $\alpha$ helix structure of the CaM-binding domain. Our data showed that reducing the mass of the selected amino acid could alter the capacity of CCaMK to interact with CaM. The mutant W342F showed an increase of CaM-binding in comparison to CCaMK, in addition, mutations W342L and W342I were able to interact with CaM but at lower levels. The mutant W342V did not interact with CaM. Interestingly, W342A mutant was able to bind to CaM similar to wild-type CCaMK even though its mass is relatively lower than that of the valine residue. Therefore, there is no direct correlation between mass of the residue at amino acid 342 and the CCaMK's CaM-binding activity.

W342F mutant had an increase of CaM-binding in comparison to CCaMK. Hence, we postulated that the kinase activity of the mutant could be significantly affected. In vitro analysis of CCaMK revealed that autophosphorylation of wild-type CCaMK is $\mathrm{Ca}^{2+}$-dependent, and it is able to phosphorylate its substrate only in the presence of $\mathrm{Ca}^{2+}$ and CaM. In this study, we observed that W342F mutant was autophosphorylated and could phosphorylate MBP substrate in the absence of $\mathrm{Ca}^{2+}$ and CaM. This strongly suggests that the activity of $\mathrm{W} 342 \mathrm{~F}$ is $\mathrm{Ca}^{2+}$ - and CaM-independent. Even though W342F was able to phosphorylate $\mathrm{MBP}$ in vitro in the presence of $\mathrm{Ca}^{2+}$ and $\mathrm{CaM}$, but the mutant barely responded to $\mathrm{Ca}^{2+}$ and $\mathrm{CaM}$ stimulation, the intensity of maximum level of MBP phosphorylation was reduced in comparison to that catalyzed by wild-type CCaMK. These results clearly indicate that the CaM-binding domain is critical for the activation of CCaMK. Also, they suggest that CCaMK protein structure may be critical for its regulation because of the possible structural changes when $\mathrm{Ca}^{2+}$ and CaM bind. The proximity of W342 to two well-known autophosphorylation sites S343 and S344 might suggest that of Trp-342 could possibly play a role during the autophosphorylation of these two sites (Routray et al., 2013; Jauregui et al., 2017).

Our physiological results demonstrated that the W342F mutation has a negative effect on the nodule morphology, since a significant portion of the nodules observed in the ccamk-1 $+\mathrm{W} 342 \mathrm{~F}$ plants were abnormal. These abnormal nodules were smaller and appeared to be infected with fewer rhizobia. This may correlate with the compromised substrate phosphorylation. The W342F mutant exhibited substrate phosphorylation in both the presence and absence of $\mathrm{Ca}^{2+}$ and $\mathrm{CaM}$, but the intensity of the phosphorylation was less than that of the wild-type in the presence of $\mathrm{Ca}^{2+}$ and CaM. This suggests that even though this mutant could be activated in the absence and presence of $\mathrm{Ca}^{2+}$ or CaM, it may not be able to sufficiently phosphorylate the target protein in order to obtain a well-developed, mature nodule in the root. In addition, our mutants did not produce any spontaneous nodules in the absence of bacteria (data not shown). This suggests that enhancing autophosphorylation and substrate phosphorylation in the absence of $\mathrm{Ca}^{2+}$ and CaM does not result in an "autoactive" kinase that can stimulate a pathway toward spontaneous nodulation. Therefore, we hypothesize that a threshold of kinase activity must be reached and this could 
trigger the production of normal, mature nodules. Since our mutant showed some reduction in the intensity of substrate phosphorylation in presence of $\mathrm{Ca}^{2+}$ and $\mathrm{CaM}$, this suggests that phosphorylation may not reach the putative threshold. Hence, the development of many nodules cannot reach the mature stage. Another possibility is that the stability of W342F mutation could be compromised when expressed in the roots.

This study documents that altering the autoinhibitory domain of CCaMK can significant increase the CaM-binding capacity of CCaMK which leads to a negative impact on root nodule development. Understanding of some target proteins of CCaMK involved in the regulation of nodule development might help to understand how CCaMK is involved not only for decoding $\mathrm{Ca}^{2+}$ oscillation but also in regulating some down-stream targets of root nodule symbiosis.

\section{AUTHOR CONTRIBUTIONS}

EJ conceived the idea for this project in consultation with BP and LD, performed most of the experiments, analyzed results and wrote most of the paper. LD and CG advised, helped in data analysis and supported with further ideas and experiments for the project. BP provided financial support, helped in planning,

\section{REFERENCES}

Antolín-Llovera, M., Ried, M. K., Binder, A., and Parniske, M. (2012). Receptor kinase signaling pathways in plant-microbe interactions. Annu. Rev. Phytopathol. 50, 451-473. doi: 10.1146/annurev-phyto-081211173002

Du, L., Ali, G. S., Simons, K. A., Hou, J., Yang, T., Reddy, A. S. N., et al. (2009). $\mathrm{Ca}^{2+} /$ calmodulin regulates salicylic-acid-mediated plant immunity. Nature 457, 1154-1158. doi: 10.1038/nature07612

Du, L., and Poovaiah, B. W. (2005). $\mathrm{Ca}^{2+} /$ calmodulin is critical for brassinosteroid biosynthesis and plant growth. Nature 437, 741-745. doi: 10.1038/nature07612

Gifford, J. L., Jamshidiha, M., Mo, J., Ishida, H., and Vogel, H. J. (2013). Comparing the calcium binding abilities of two soybean calmodulins: towards understanding the divergent nature of plant calmodulins. Plant Cell 25, 4512-4524. doi: 10.1105/tpc.113.113183

Gleason, C., Chaudhuri, S., Yang, T., Muñoz, A., Poovaiah, B. W., and Oldroyd, G. E. D. (2006). Nodulation independent of rhizobia induced by a calciumactivated kinase lacking autoinhibition. Nature 441, 1149-1152. doi: 10.1038/ nature 04812

Harper, J. F., Breton, G., and Harmon, A. (2004). Decoding $\mathrm{Ca}^{2+}$ signals through plant protein kinases. Annu. Rev. Plant Biol. 55, 263-288. doi: 10.1146/annurev. arplant.55.031903.141627

Hayashi, T., Banba, M., Shimoda, Y., Kouchi, H., Hayashi, M., and Imaizumi-Anraku, H. (2010). A dominant function of CCaMK in intracellular accommodation of bacterial and fungal endosymbionts. Plant J. 63, 141-154. doi: 10.1111/j.1365-313X.2010. 04228.x

Horváth, B., Yeun, L. H., Domonkos, A., Halász, G., Gobbato, E., Ayaydin, F., et al. (2011). Medicago truncatula IPD3 is a member of the common symbiotic signaling pathway required for rhizobial and mycorrhizal symbioses. Mol. Plant. Microbe Interact. 24, 1345-1358. doi: 10.1094/MPMI-01-110015

Jang, D. J., Ban, B., and Lee, J. A. (2011). Characterization of novel calmodulin binding domains within IQ motifs of IQGAP1. Mol. Cells 32, 511-518. doi: 10.1007/s10059-011-0109-4

Jauregui, E., Du, L., Gleason, C., and Poovaiah, B. W. (2017). Autophosphorylation of calcium/calmodulin-dependent protein kinase (CCaMK) at S343 executing and leading the project. EJ, LD, CG, and BP were involved in manuscript preparation.

\section{FUNDING}

This research was supported by the National Science Foundation grant (1021344), and the Washington State University Agricultural Research Center.

\section{ACKNOWLEDGMENTS}

The authors thank Prof. R. Geurts for sharing the Sinorhizobium meliloti strain. The authors also acknowledge the WSU Franceschi Microscopy and Imaging Center for assistance with the Confocal microscopy. The authors also thank Lorie Mochel for her help in editing.

\section{SUPPLEMENTARY MATERIAL}

The Supplementary Material for this article can be found online at: https://www.frontiersin.org/articles/10.3389/fpls.2017.01921/ full\#supplementary-material

or S344 generates an intramolecular interaction blocking the CaMbinding. Plant Signal. Behav. 12, e1343779. doi: 10.1080/15592324.2017. 1343779

Jin, Y., Liu, H., Luo, D., Yu, N., Dong, W., Wang, C., et al. (2016). DELLA proteins are common components of symbiotic rhizobial and mycorrhizal signaling pathways. Nat. Commun. 7:12433. doi: 10.1038/ncomms 12433

Lévy, J., Bres, C., Geurts, R., Chalhoul, B., Kulikova, O., Gérard, D., et al. (2004). A putative $\mathrm{Ca}^{2+}$ and calmodulin-dependent protein kinase required for bacterial and fungal symbioses. Science 303, 1361-1364. doi: 10.1126/science. 1093038

Liu, H., and Naismith, J. H. (2008). An efficient one-step site-directed deletion, insertion, single and multiple-site plasmid mutagenesis protocol. BMC Biotechnol. 8:91. doi: 10.1186/1472-6750-8-91

Liu, Y., Zheng, X., Mueller, G. A., Sobhany, M., DeRose, E., Zhang, Y., et al. (2012). Crystal structure of calmodulin binding domain of Orail in complex with $\mathrm{Ca}^{2+} /$ calmodulin displays a unique binding mode. J. Biol. Chem. 287, 43030-43041. doi: 10.1074/jbc.M112.380964

Miller, J. B., Pratap, A., Miyahara, A., Zhou, L., Bornemann, S., Morris, R. J., et al. (2013). Calcium/calmodulin-dependent protein kinase is negatively and positively regulated by calcium, providing a mechanism for decoding calcium responses during symbiosis signaling. Plant Cell 25, 5053-5066. doi: 10.1105/ tpc.113.116921

Nanjareddy, K., Blanco, L., Arthikala, M.-K., Alvarado-Affantranger, X., Quinto, C., Sánchez, F., et al. (2016). A legume TOR protein kinase regulates rhizobium symbiosis and is essential for infection and nodule development. Plant Phys. 172, 2002-2020. doi: 10.1104/pp.16.00844

Parniske, M. (2004). Molecular genetics of the arbuscular mycorrhizal symbiosis. Curr. Opin. Plant Biol. 7, 414-421. doi: 10.1016/j.pbi.2004.05.011

Patil, S., Takezawa, D., and Poovaiah, B. W. (1995). Chimeric plant calcium/calmodulin-dependent protein kinase gene with a neural visinin-like calcium-binding domain. Proc. Natl. Acad. Sci. U.S.A. 92, 4897-4901.

Peng, Z., Liu, F., Wang, L., Zhou, H., Paudel, D., Tan, L., et al. (2017). Transcriptome profiles reveal gene regulation of peanut (Arachis hypogaea L.) nodulation. Sci. Rep. 7:40066. doi: 10.1038/srep40066

Poovaiah, B. W., Du, L., Wang, H., and Yang, T. (2013). Recent advances in calcium/calmodulin-mediated signaling with an emphasis on plant-microbe interactions. Plant Physiol. 163, 531-542. doi: 10.1104/pp.113.220780 
Pumplin, N., Mondo, S. J., Topp, S., Starker, C. G., Gantt, J. S., and Harrison, M. J. (2010). Medicago truncatula Vapyrin is a novel protein required for arbuscular mycorrhizal symbiosis. Plant J. 61, 482-494. doi: 10.1111/j.1365-313X.2009. 04072.x

Ramachandiran, S., Takezawa, D., Wang, W., and Poovaiah, B. W. (1997). Functional domains of plant chimeric calcium/calmodulin-dependent protein kinase: regulation by autoinhibitory and visinin-like domains. J. Biochem. 121, 984-990. doi: 10.1093/oxfordjournals.jbchem.a021684

Routray, P., Miller, J. B., Du, L., Oldroyd, G., and Poovaiah, B. W. (2013). Phosphorylation of S344 in the calmodulin-binding domain negatively affects CCaMK function during bacterial and fungal symbioses. Plant J. 76, 287-296. doi: $10.1111 /$ tpj.12288

Sathyanarayanan, P. V., and Poovaiah, B. W. (2002). Autophosphorylationdependent inactivation of plant chimeric calcium/calmodulin-dependent protein kinase. Eur. J. Biochem. 269, 2457-2463. doi: 10.1046/j.1432-1033.2002. 02904.x

Sathyanarayanan, P. V., Siems, W. F., Jones, J. P., and Poovaiah, B. W. (2001). Calcium-stimulated autophosphorylation site of plant chimeric calcium/calmodulin-dependent protein kinase. J. Biol. Chem. 276, 32940-32947. doi: 10.1074/jbc.M009648200

Shimoda, Y., Han, L., Yamazaki, T., Suzuki, R., Hayashi, M., and ImaizumiAnraku, H. (2012). Rhizobial and fungal symbioses show different requirements for calmodulin binding to calcium calmodulin-dependent protein kinase in Lotus japonicus. Plant Cell 24, 304-321. doi: 10.1105/tpc.111.092197

Singh, S., and Parniske, M. (2012). Activation of calcium- and calmodulindependent protein kinase (CCaMK), the central regulator of plant root endosymbiosis. Curr. Opin. Plant Biol. 15, 444-453. doi: 10.1016/j.pbi.2012. 04.002

Snedden, W. A., and Fromm, H. (2001). Calmodulin as a versatile calcium signal transducer in plants. New Phytol. 125, 35-66. doi: 10.1046/j.1469-8137.2001. 00154.x

Takeda, N., Maekawa, T., and Hayashi, M. (2012). Nuclear-localized and deregulated calcium- and calmodulin-dependent protein kinase activates rhizobial and mycorrhizal responses in Lotus japonicus. Plant Cell 24, 810-822. doi: 10.1105/tpc.111.091827

Takezawa, D., Ramachandiran, S., Paranjape, V., and Poovaiah, B. W. (1996). Dual regulation of a chimeric plant serine/threonine kinase by calcium and calcium/calmodulin. J. Biol. Chem. 271, 8126-8132. doi: 10.1074/jbc.271.14. 8126

Tidow, H., and Nissen, P. (2013). Structural diversity of calmodulin binding to its target sites. FEBS J. 280, 5551-5565. doi: 10.1111/febs.12296

Tirichine, L., Imaizumi-Anraku, H., Yoshida, S., Murakami, Y., Madsen, L. H., Miwa, H., et al. (2006). Deregulation of a $\mathrm{Ca}^{2+} /$ calmodulin-dependent kinase leads to spontaneous nodule development. Nature 441, 1153-1156. doi: $10.1038 /$ nature 04862

Wang, J.-P., Munyampundu, J.-P., Xu, Y.-P., and Cai, X.-Z. (2015). Phylogeny of plant calcium and calmodulin-dependent protein kinases (CCaMKs) and functional analyses of tomato CCaMK in disease resistance. Front. Plant Sci. 6:1075. doi: 10.3389/fpls.2015.01075

Wang, W., and Poovaiah, B. W. (1999). Interaction of plant chimeric calcium/calmodulin-dependent protein kinase with a homolog of eukaryotic elongation factor-1. J. Biol. Chem. 274, 12001-12008. doi: 10.1074/jbc.274.17. 12001

Yang, T., Du, L., and Poovaiah, B. W. (2007). Concept of redesigning proteins by manipulating calcium/calmodulin-binding domains to engineer plants with altered traits. Funct. Plant Biol. 34, 343. doi: 10.1071/FP06293

Yano, K., Yoshida, S., Müller, J., Singh, S., Banba, M., Vickers, K., et al. (2008). CYCLOPS, a mediator of symbiotic intracellular accommodation. Proc. Natl. Acad. Sci. U.S.A. 105, 20540-20545. doi: 10.1073/pnas.08068 58105

Yuan, P., Jauregui, E., Du, L., Tanaka, K., and Poovaiah, B. W. (2017). Calcium signatures and signaling events orchestrate plant-microbe interactions. Curr. Opin Plant Biol. 38, 173-183. doi: 10.1016/j.pbi.2017.06.003

Conflict of Interest Statement: The authors declare that the research was conducted in the absence of any commercial or financial relationships that could be construed as a potential conflict of interest.

Copyright (C) 2017 Jauregui, Du, Gleason and Poovaiah. This is an open-access article distributed under the terms of the Creative Commons Attribution License (CC BY). The use, distribution or reproduction in other forums is permitted, provided the original author(s) or licensor are credited and that the original publication in this journal is cited, in accordance with accepted academic practice. No use, distribution or reproduction is permitted which does not comply with these terms. 